\title{
A Study on the Sloshing Phenomena of Membrane type LNG Carrier in the Time Domain
}

\author{
by Pu-Keun Paik*, Mun-Keun Ha*, Member, Mun-Seung Kim* \\ Shin-Yung Kang**
}

\begin{abstract}
Summary
Sloshing in the liquefied natural gas $(\mathrm{LNG})$ carrier occur due to the large dimension of the tank and the characteristics of the liquefied cargo. The description of nonlinear fluid flow and random tank motion in irregular waves are required to predict the sloshing phenomena. A numerical code adopting SOLA-VOF scheme has been developed to analyse sloshing flow. For the ship motion analysis 3D panel method is applied and the results are compared with those of ABS class. Furthermore, to evaluate sloshing load in the directional waves, this study used ISSC directional wave spectrum with cosine square directional spreading function. Ship motion time histories are used as the input data of sloshing calculation. We performed sloshing analysis for No.2 tank of $138 \mathrm{~K}$ LNG carrier with fully loaded condition. Higher sloshing responses are predicted on head sea where the sloshing peak pressures are inversely proportional to the zero crossing wave periods than the other seas in high filling and severe sea conditions. The calculated maximum peak pressures considering the cushioning effects of corrugated membrane in head sea can be used as the external forces for the structural analysis.
\end{abstract}

\section{Introduction}

The flow characteristics of sloshing in the tank is determined by the dimension, filling height, tank motion period and amplitude. To keep the difference between the motion and resonance period large, the fluids in the tank have to continue linear motion. Howerver, as the difference of the periods approaches to zero, the flow in the tank shows nonlinear motion similar to as hydraulic jump, wave breaking and large impact force may occur on the wall or ceiling. The damages of LNG tank and cargo holder of large crude oil tanker by the sloshing impact load have been reported occasionally ${ }^{1)}$.

The tanks of VLCC and LNG carrier have very large size and subject to sloshing problem. Nowaday evaluation of sloshing load is motivated and emphasized because of the increasing demands of larger LNG carrier and long time moored FPSO tankers. The sloshing analysis for LNG carrier is required for structural design in the cargo hold to prevent brittle fracture and fatigue collapse of the structure inside tank such as a pump tower. The extreme maximum load of sloshing in the tank is needed for the structural analysis. ISSC ${ }^{2)}$ also promoted that sloshing load has to be considered one to be important environmental load conditions in the structural analysis and summarized the state of art in

* Samsung Heavy Industries, Ltd.

** Korea Maritime University

Received 10 th July 1998

Read at the Autumn meeting 12, 13 th Nov. 1998 the study on the sloshing.

For assessing sloshing effects, the experimental and numerical analysis are the general tools to ship designers. The numerical results of the sloshing simulation have been compared with the experimental results using the 2 dimensional model ${ }^{3), 4), 5), 6)}$ and 3 dimensional model ${ }^{7), 8)}$. Rizzuto and Tedesh ${ }^{9}$ ) showed the comparison results between the numerical calculations and full scale pressure measurement at the ballast tank of a bulk carrier.

In the numerical calculation $2 \mathrm{D}$ method (e.g. LRFLUIDS, ABS-SLOSH) has been practically used to ship designer. SOLA-SURF code ${ }^{10)}$ uses the height function to estimate the free surface and is useful for the case when the free surface can be expressed with single valued function. In spite of this deficiency many researchers have been developed the SURF scheme ${ }^{11), 12), 13)}$. Nichols et al. ${ }^{14)}$ had developed the method of Volume of Fluid(VOF) and described the free surface with the fluid possession rate of defined mesh. Then the VOF code is useful at the nonlinear free surface such as braking wave or overturning flow in the $\operatorname{tank}^{15), 16)}$. Due to this reason, a $3 \mathrm{D}$ VOF scheme is developed for the sloshing analysis of $138 \mathrm{~K}$ LNG carrier in this study.

For the practical sloshing calculation, the ship motion has to be calculated in the time domain. Chun et al. ${ }^{17)}$ has developed the 3 dimensional numerical scheme based on the source distribution method and 
applied the scheme to high speed coastal passenger SWATH ship. Kim ${ }^{17)}$ tested the program for the calculation of the motion RAO of single hull $138 \mathrm{~K} \mathrm{LNG}$ carrier. These estimated motion RAO can be transformed to the motion time history as the input data for the sloshing simulation.

\section{Formulation of Sloshing Problem}

\subsection{Governing equation}

The continuity and Navier-Stokes equations for an incompressible fluid are the governing equations for the Volume of Fluid(VOF) method ${ }^{18)}$ for a three dimensional flow. The equations are described as follows,

$$
\begin{aligned}
& \nabla \cdot \mathbf{V}=0 \\
& \frac{d \mathbf{V}}{d t}=-\frac{1}{\rho} \nabla p+\frac{\mathbf{f}}{\rho}+\nu \nabla^{2} \mathbf{V}
\end{aligned}
$$

where $\mathbf{V}$ is velocity vector of fluid particles, $\mathbf{f}$ represents body force, $\rho$ is the density of fluids, and $\nu$ is the kinematic viscosity coefficient.

\subsection{Body forces in moving coordinate}

Figure 1 shows the three coordinate systems to express the body forces. The coordinate $O-X_{1} X_{2} X_{3}$ means the fixed coordinate in space(moving coordinate) to describe Newtonian law. $\bar{o}-y_{1} y_{2} y_{3}$ and $o-x_{1} x_{2} x_{3}$ coordinates are fixed to the moving tank. If three observers at $O, \bar{o}$ and $o$ observe the one point $M$ of the fluids within the tank at the same time, following relation between $O$ and $\bar{o}$ will be produced,

$$
X_{i}=R_{i}+F_{i, j} y_{j}
$$

where $F$ is the direction cosine between $O-X_{1} X_{2} X_{3}$ and $\bar{o}-y_{1} y_{2} y_{3}$ coordinate. The velocity and accleration of fluid $M$ which is observed by $\bar{o}$ can be obtained from eq.(3). To find the velocity and accleration of fluid $M$ observed by $o$, the transformation of coordinate is needed again. Because $\bar{o}-y_{1} y_{2} y_{3}$ and $o-x_{1} x_{2} x_{3}$ coordinates use the same basis, following realtion is obtained.

$$
y_{j}=d_{j}+x_{j}
$$

The position vector $d_{j}$ which expresses the position from the center of ship to the tank origin is independent of time. Thus, the acceleration and body force of fluid $M$ which is observed by $o$ can be found from eq. (4)

$$
\begin{aligned}
a_{k} & =Q_{k i}\left(A_{i}-B_{i}\right)+f_{k} \\
f_{k} & =Q_{k i}\left(B_{i}-\ddot{R}_{i}\right)-Q_{k i} F_{i j}\left(x_{j}+d_{j}\right) \\
& -2 Q_{k i} \dot{F}_{i j} u_{j}
\end{aligned}
$$

where the $a_{k}$ and $f_{k}$ are the acceleration and the body force of fluid $M$ respectively. $Q$ means the linear transformation from $O-X_{1} X_{2} X_{3}$ to $\bar{o}-y_{1} y_{2} y_{3}$ coordinate and orthogonal to $F . A_{i}$ and $B_{i}$ are the acceleration of fluid observed by $O$ and the acceleration of gravity respectively.

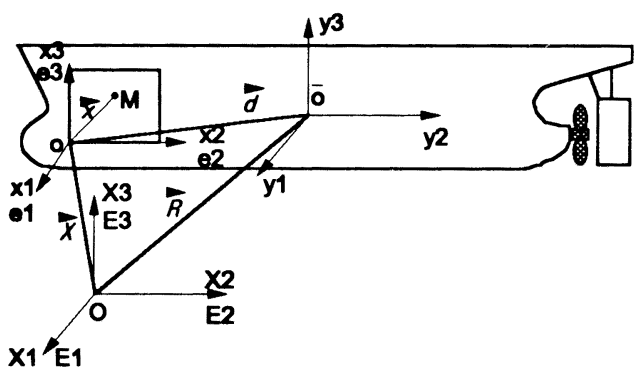

Fig.1 Coordinate systems

When the component of each coordinate axis is expressed to the engineering notation $(x, y, z$ instead of $x_{1}, x_{2}, x_{3}$;subscripts $x, y, z$ instead of subscripts $1,2,3$ ), the body forces considering the ship motion of 6 degrees of freedom(DOF) are following ,

$$
\begin{aligned}
f_{x} & =\left(B_{x}-\ddot{R}_{x}\right) \cos \theta_{y} \cos \theta_{z} \\
& +\left(B_{y}-\ddot{R}_{y}\right) \cos \theta_{x} \sin \theta_{z} \\
& +\left(B_{y}-\ddot{R}_{y}\right) \sin \theta_{x} \sin \theta_{y} \cos \theta_{z} \\
& +\left(B_{z}-\ddot{R}_{z}\right) \sin \theta_{x} \sin \theta_{z} \\
& -\left(B_{z}-\ddot{R}_{z}\right) \cos \theta_{x} \sin \theta_{y} \cos \theta_{z} \\
& +\omega_{x}\left(y+d_{y}\right) \sin \theta_{y} \\
& +\omega_{x}\left(z+d_{z}\right) \cos \theta_{y} \sin \theta_{z} \\
& -\omega_{y}\left(z+d_{z}\right) \cos \theta_{z}+\dot{\omega}_{z}\left(y+d_{y}\right) \\
& +\omega_{y}^{2}\left(x+d_{x}\right)+\omega_{z}^{2}\left(x+d_{x}\right) \\
& -2 \omega_{x} \omega_{y}\left(x+d_{x}\right) \cos \theta_{y} \sin \theta_{z} \cos \theta_{z} \\
& +2 \omega_{x} \omega_{y}\left(y+d_{y}\right) \cos \theta_{y} \sin \theta_{z}^{2} \\
& -2 \omega_{x} \omega_{y}\left(z+d_{z}\right) \sin \theta_{y} \sin \theta_{z} \\
& +2 \omega_{x} \omega_{y}\left(x+d_{x}\right) \sin \theta_{y} \\
& +2 \omega_{x} v \sin \theta_{y}+2 \omega_{x} w \cos \theta_{y} \sin \theta_{z} \\
& -2 \omega_{y} w \cos \theta_{z}+2 \omega_{z} v \\
& \\
& =\left(B_{x}-\ddot{R_{x}}\right) \cos \theta_{y} \sin \theta_{z} \\
+ & \left(B_{y}-\ddot{R}_{y}\right) \cos \theta_{x} \cos \theta_{z} \\
f_{y} &
\end{aligned}
$$




$$
\begin{aligned}
& +\quad\left(B_{y}-\ddot{R}_{y}\right) \sin \theta_{x} \sin \theta_{y} \sin \theta_{z} \\
& +\quad\left(B_{z}-\ddot{R}_{z}\right) \sin \theta_{x} \cos \theta_{z} \\
& -\quad\left(B_{z}-\ddot{R}_{z}\right) \cos \theta_{x} \sin \theta_{y} \sin \theta_{z} \\
& +\omega_{x}\left(x+d_{x}\right) \sin \theta_{y} \\
& +\quad \omega_{x}\left(z+d_{z}\right) \cos \theta_{y} \cos \theta_{z} \\
& -\quad \omega_{y}\left(z+d_{z}\right) \sin \theta_{z}-\dot{\omega}_{z}\left(x+d_{x}\right) \\
& +\quad \omega_{x}^{2}\left(y+d_{y}\right)+\omega_{z}^{2}\left(y+d_{y}\right) \\
& -\quad 2 \omega_{x} \omega_{y}\left(x+d_{x}\right) \cos \theta_{y} \cos \theta_{z}^{2} \\
& +2 \omega_{x} \omega_{y}\left(y+d_{y}\right) \cos \theta_{y} \sin \theta_{z} \cos \theta_{z} \\
& -2 \omega_{x} \omega_{y}\left(z+d_{z}\right) \sin \theta_{y} \cos \theta_{z} \\
& +2 \omega_{x} \omega_{z}\left(y+d_{y}\right) \sin \theta_{y} \\
& -2 \omega_{x} u \sin \theta_{y}+2 \omega_{x} w \cos \theta_{y} \cos \theta_{z} \\
& +2 \omega_{y} w \sin \theta_{z}-2 \omega_{z} u
\end{aligned}
$$

$$
\begin{aligned}
f_{z} & =\left(B_{x}-\ddot{R}_{x}\right) \sin \theta_{y} \\
& -\left(B_{y}-\ddot{R}_{y}\right) \sin \theta_{x} \cos \theta_{y} \\
& +\left(B_{z}-\ddot{R}_{z}\right) \cos \theta_{x} \cos \theta_{y} \\
& -\omega_{x}\left(x+d_{x}\right) \cos \theta_{y} \sin \theta_{z} \\
& -\omega_{x}\left(y+d_{y}\right) \cos \theta_{y} \cos \theta_{z} \\
& -\omega_{y}\left(x+d_{x}\right) \cos \theta_{z}-\dot{\omega}_{y}\left(y+d_{y}\right) \sin \theta_{z} \\
& +\omega_{x}^{2}\left(z+d_{z}\right)+\omega_{y}^{2}\left(z+d_{z}\right) \\
& -2 \omega_{z} \omega_{x}\left(x+d_{x}\right) \cos \theta_{y} \cos \theta_{z} \\
& +2 \omega_{x} \omega_{z}\left(y+d_{y}\right) \cos \theta_{y} \sin \theta_{z} \\
& -2 \omega_{z} \omega_{y}\left(x+d_{x}\right) \sin \theta_{z} \\
& -2 \omega_{z} \omega_{y}\left(y+d_{y}\right) \cos \theta_{z} \\
& -2 \omega_{x} u \cos \theta_{y} \sin \theta_{z}-2 \omega_{x} v \cos \theta_{y} \cos \theta_{z} \\
& +2 \omega_{y} u \cos \theta_{z}-2 \omega_{y} v \sin \theta_{z}
\end{aligned}
$$

where $B_{x}, B_{y}$ and $B_{z}$ are the acceleration components of gravity in $\mathrm{x}, \mathrm{y}$ and $\mathrm{z}$ direction respectively, $\ddot{R}_{x}, \ddot{R_{y}}$ and $\ddot{R_{z}}$ are added inertial force components due to ship motion, and $\theta_{x}, \theta_{y}, \theta_{z}$ are the amplitudes of roll, pitch, yaw. $\omega_{x}, \omega_{y}, \omega_{z}$ are angular velocities of roll, pitch, yaw, $\dot{\omega}_{x}, \dot{\omega}_{y}, \dot{\omega}_{z}$ are angular accelerations, $d_{x}, d_{y}, d_{z}$ are the position components of the distance between the origin of tank and the center of gravity.

\subsection{Boundary condition}

The boundary of fluids means that the fluids can flow within the boundary. The fluids cannot flow through the boundary like the free surface or solid surface, which is the kinematical boundary condition. The boundary of fluids is expressed as $\Omega(x, y, z, t)=0$ and the kinematical boundary condition at the boundary is $D \Omega / D t=0$. The dynamical boundary condition at the free surface satisfies following boundary conditon,

$$
p=p_{\circ}+2 \mu \frac{\partial u_{n}}{\partial n} \quad \text { on } \quad \Omega(x, y, z, t)=0
$$

where $p_{0}$ is the the pressue of atmosphere, $\mu$ is the coefficient of viscosity, $n$ is the normal vector on the free surface. It is necessary to set conditions at all mesh boundaries and at surfaces of all internal obstacles. At the mesh boundaries, a variety of conditions may be set using the layer of fictitious cells surrounding the mesh. For example, if the left boundary is a rigid free slip wall, the normal velocity there must be zero and the tangential velocity should have normal gradient,

$$
u_{1, j, k}=0, \quad v_{1, j, k}=v_{2, j, k}, \quad w_{1, j, k}=w_{2, j, k}
$$

In the case of internal obstacles that are restricted to shapes all velocity components within obstacles are set to zero. That is, tangential velocities are zero at locations shifted from the actual boundary by one-half of a cell width. Especially, for the sloping wall cell pressures are iteratively adjusted by eq.(2 ) and eq.(11) until eq.(1) is satisfied to a prespecified level of accuracy and following pressure equation is satisfied.

$$
\Delta p=-\frac{U_{n}}{\Delta t} \frac{\Delta x \Delta y}{\sqrt{\Delta x^{2} \Delta y^{2}}}
$$

The advancement of the volume of fluids in time is carried out by a doner-acceptor model ${ }^{19)}$ in the SOLAVOF. Thus, the equations of continuity are expressed differently at the each sloping wall of corner.

\section{Numerical Method}

\subsection{Finite difference equations}

In the case of 2 dimensional SOLA-VOF, Hirt et $a l^{18)}$ and Nichols et $a l^{14)}$ dealt with the discretization of equations, the boundary condition, the treatment of the free surface and the algorithm of fluid flow transportation. Su et $\left.a^{19}\right)^{19}$ treated the application of VOF to sloshing problem in detail. 3 dimensional equations can be developed from the difference equations of 2 dimensional SOLA-VOF algorithm. The discretization of the momentum eq.(2) are expressed using the subscripts $i, j$ and $k$ for the space and the superscripts $n$ for the time,

$$
\begin{aligned}
u_{i, j, k}^{n+1} & =u_{i, j, k}^{n}+\Delta t\left[\frac { 1 } { \Delta x } \left(P_{i, j, k}^{n}-P_{i+1, j, k}^{n}\right.\right. \\
& +B_{x}-F U X-F U Y-F U Z \\
& +V I X) \\
v_{i, j, k}^{n+1} & =v_{i, j, k}^{n}+\Delta t\left[\frac { 1 } { \Delta y } \left(P_{i, j, k}^{n}-P_{i, j+1, k}^{n}\right.\right. \\
& +B_{y}-F V X-F V Y-F V Z \\
& +V I Y) \\
w_{i, j, k}^{n+1} & =w_{i, j, k}^{n}+\Delta t\left[\frac { 1 } { \Delta z } \left(P_{i, j, k}^{n}-P_{i, j, k+1}^{n}\right.\right.
\end{aligned}
$$




$$
\begin{aligned}
& +B_{z}-F W X-F W Y-F W Z \\
& +\quad V I Z)
\end{aligned}
$$

$F U X, F U Y, F U Z, F V X, F V Y, F V Z, F W X, F W Y$, $F W Z$ mean the advection terms and $V I X, V I Y, V I Z$ are the viscosity terms. $\Delta x, \Delta y$ and $\Delta z$ are grid spaces and $\Delta t$ is the time increment.

\subsection{Solution procedure}

The velocity values don't satisfy the continuity equation generally. The velocity and pressure are adjusted iteratively as following procedure,

1) Calculate the divergence $D$ using the calculated velocities, $u, v, w$

$$
\begin{aligned}
D & =\frac{1}{\Delta x}\left[u_{i, j, k}^{n+1}-u_{i-1, j, k}^{n+1}\right] \\
& +\frac{1}{\Delta y}\left[v_{i, j, k}^{n+1}-v_{i, j-1, k}^{n+1}\right] \\
& +\frac{1}{\Delta z}\left[w_{i, j, k}^{n+1}-w_{i, j, k-1}^{n+1}\right]
\end{aligned}
$$

2) After find $\Delta p$ from eq.(16), adjust the velocities at the boundary.

3) Calculate eq.(16) again from the adjusted velocity field $(u, v, w)$.

4) Repeat the procedures 1) 3) until the result of eq.(16) satisfies the allowable level of error.

The integral calculus for the velocity and pressure goes on until the prespecified time.

\subsection{Boundary condition}

The locating of free surface is difficult as one simulates a free surface. To treat the free surface a function $F(x, y, z, t)$ that is defined to be unity at any point occupied by the fluid and zero elsewhere is introduced. $F=1$ implies a cell full of fluid, while $F=0$ means an empty cell. Cells with $F$ value between zero and unity are partially filled with fluid. A free surface cell contains a non-zero value of $F$ and at least one neighboring cell contains zero value of $F$.

It is not easy to treat the free surface boundary condition numerically. In this study, the free surface boundary condition was dealt with as follows.

(1) Calculate the slope for the horizontal plane and the vertical plane approximately from the neighboring $F$ values.

(2) Find the shape of fluids from the $F$ values and slope, and determine the cell which is very near the free surface to correct the pressure.

(3) Calculate the pressure of the free surface using the distance between the center of selected cell and the free surface by means of the linear interpolation to make the pressure balance between the atmosphere and the free surface.

(4) Adjust the velocities of the neighboring cells from the calculated pressure and make the neighboring cells satisfy eq.(1) iteratively.

\section{Comparative Study}

To verify the developed code for the sloshing analysis we performed first series of simulations for $2 \mathrm{D}$ model. The experimental results of Arai ${ }^{11)}$ were compared with the calculation results of ours using the developed code.

In $2 \mathrm{D}$ case, we could verify the code for the model tank with no upper chamfers and no baffles. The length of tank is $0.6 \mathrm{~m}$, heght is $0.4 \mathrm{~m}$ and filling ratio is $50 \%$. The excitation was the sway motion of 1.0 $\mathrm{Hz}$ frequency and $5 \mathrm{~mm}$ amplitude. The mesh system of $48 \times 32$ elements was adopted for simulation. The height of the free suface and the pressures at 4 positions were compared with the experimental results of Arai. Figure 2(a) and Fig.2(b) show that the calculation results of the developed code and the results of Arai, simultaneously. The comparisons result in good agreement, qualitively and quantitively.

In $3 \mathrm{D}$ case, we compared the results of Arai with our results of $3 \mathrm{D}$ calculation. The $3 \mathrm{D}$ model tank doesn't also have upper chamfers and baffles in the tank. The dimension of tank is $0.6 \times 0.6 \times 0.125 \mathrm{~m}$ and the filling ratio is $50 \%$. The frequency and the amplitude of the sway motion are also $1.0 \mathrm{~Hz}$ and $5 \mathrm{~mm}$, respectively. The mesh system is composed of $24 \times 16 \times 5$ elements. The height of free surface and the 4 pressures like the case of $2 \mathrm{D}$ were compared with the experimental results of Arai. Fig. 2(c) shows the results of $3 \mathrm{D}$ calculation and good agreement with $2 \mathrm{D}$ experimental results.

The developed simulation methods are expected to furnish a practical and reliable means for the estimate of sloshing loads in the liquid cargo tank design from the above. The sloshing analysis for the cargo tank of LNG carrier has been performed with the developed code in this study.

\section{Sloshing in LNG carrier}

It is important to assess the sloshing loads as one of the external forces treated in the design of the tank and the internal structures of LNG carrier. The nature of sloshing impacts is highly random and nonlinear. To predict the sloshing behavior and responses accurately, random tank motions in time domain are needed to drive tank, and nonlinear fluid flow simulations are required to determine sloshing responses.

\subsection{Estimation of ship motion}

To calculate 3 dimensional ship motion we used the calculation program based on 3 dimensional diffraciton theory. Chun et al ${ }^{17}$ ) used a potential based 3D panel method to develop for the calculation of the $6 \mathrm{DOF}$ responses of a SWATH ship travelling in waves at an 


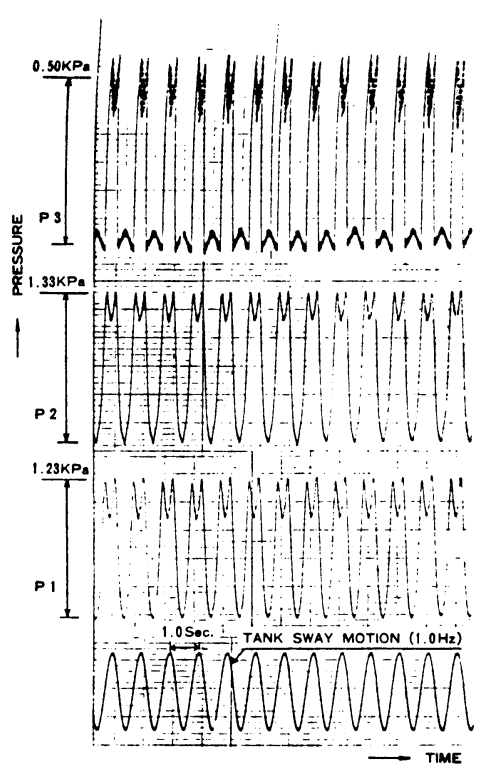

Fig.2(a) Experimental results (by Arai)
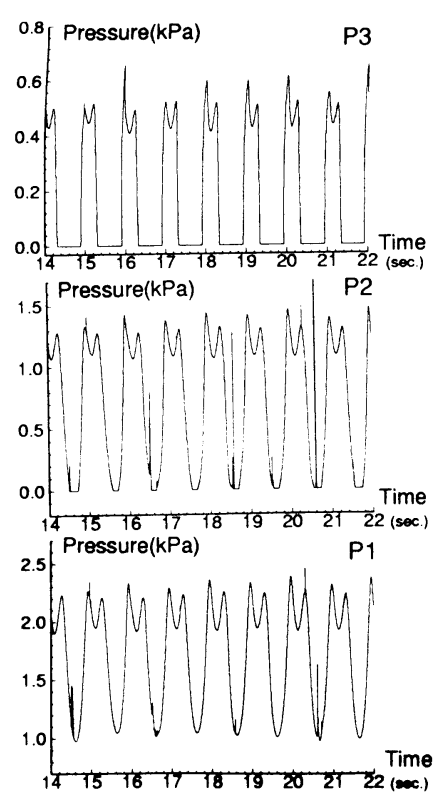

Fig.2(b) 2D calculation results
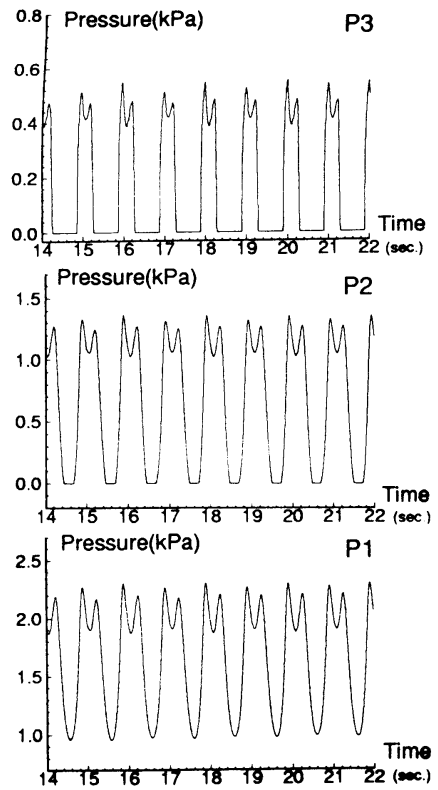

Fig.2(c) 3D calculation results arbitrary heading angle. In this study the application of the program is extended to the mono hull $138 \mathrm{~K} \mathrm{LNG}$ carrier. Potential theories are based on the ideal flow assumptions, and then attribute all damping effects to wave making damping. This theory gives proper results for all modes of motion and viscous effects on the roll motion are taken into account. The velocity potential satisfies the Laplace's equation and the boundary conditions considering the linearization of the waves. Under the assumption that the responses are linear and harmonic, 6 linear coupled differential equations of motion can be written in following form,

$$
\sum_{j=1}^{6}\left[-\omega_{e}^{2}\left(M_{i j}+A_{i j}\right)-i \omega_{e} B_{i j}+C_{i j}\right] \xi_{j}=\bar{F}_{i}
$$

where $i, j=1 \sim 6, M_{i j}$ is the generalized mass matrix for the ship, $A_{i j}, B_{i j}, C_{i j}$ are the added mass, damping and restoring force matrices respectively, $\xi_{i}$ is the amplitudes of the response motion in each of six DOF, and $\bar{F}_{i}$ is the amplitudes of the wave exciting force and moment.

The principal dimension of $\mathrm{LNG}$ carrier is shown in Table 1. The ship motions are calculated on the speed conditions of $0,10.0$ and 15.225 Knots in head seas, bow quartering seas, beam seas, stern quartering seas and following seas. In these calculations the hull surface of the ship is discretized for half body because of its symmetry. Figure 3 shows the RAO of 6 DOF motion respectively in all heading angles as the ship speed is 15.225 Knots. Estimated motion results for $138 \mathrm{~K}$ LNG carrier are compared with the calculated results based on strip theory which is carried out by the class $\mathrm{ABS}^{20)}$. Heave and pitch $\mathrm{RAO}$ at $90^{\circ}$ and $120^{\circ}$ respectively is slightly larger than those of ABS, but the other motion RAOs in the regular waves show good agreement.

\subsection{Random tank motion}

The ocean going ship is actually encountering the long-crested or short-crested irregular waves depending on the Beaufort number. The actual motion of vessel is estimated from the ship motion RAO. The actual waves which the LNG carrier will operate in, is assumed to be the ITTC standard wave spectrum,

Table 1: Principal dimension of LNG carrier and No.2 $\operatorname{tank}(\mathrm{m})$

\begin{tabular}{|c|c||c|c|}
\hline LBP & 266.0 & tank length & 43.3 \\
\hline Beam overal & 42.6 & tank width & 37.4 \\
\hline Depth & 26. & tank height & 26.86 \\
\hline Draft & 11.3 & $\begin{array}{c}\text { tank } \\
\text { chamfer }\end{array}$ & $\begin{array}{c}3.9 \times 3.75 \text { (bot.) } \\
8.3 \times 8.3 \text { (top) }\end{array}$ \\
\hline
\end{tabular}



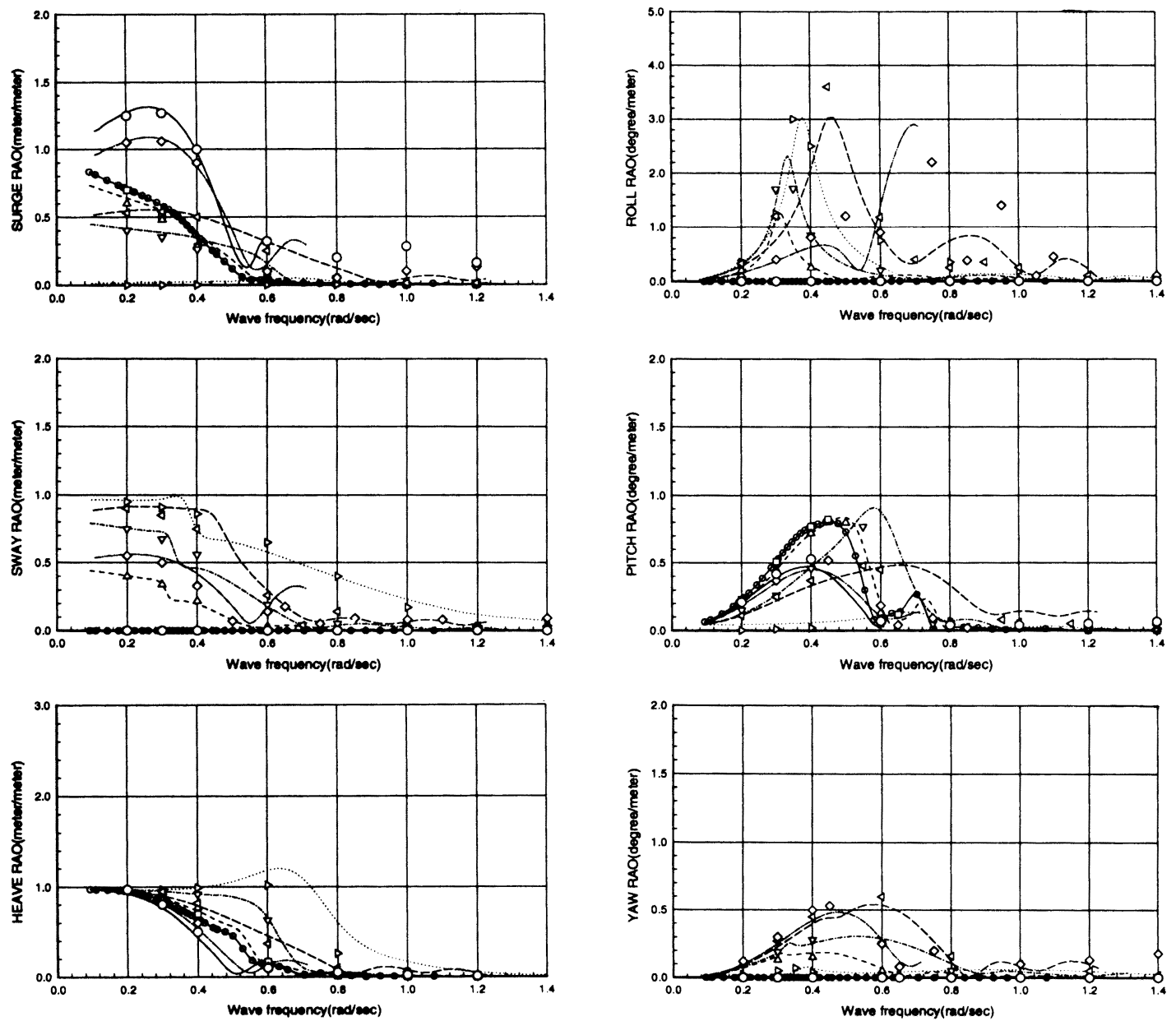

Fig.3 Motion RAOs of $138 \mathrm{~K} \mathrm{LNG}$ carrier(ship speed $=15.225 \mathrm{knots}$ )

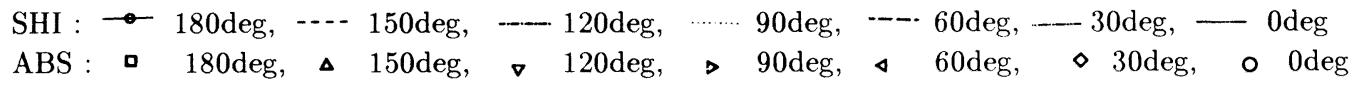

$$
S\left(\omega_{w}\right)=\frac{A}{\omega_{w} 5} e^{-B / \omega_{w}}
$$

where $A=173.0 H_{1 / 3}^{2} / T^{4}, B=691.0 / T^{4}$. When a ship with mean forward speed $V$ navigates on a sea expressed by a wave spectrum, the ship recognizes the spectrum in terms of the encountering frequencies as following,

$$
S\left(\omega_{e}\right)=S\left(\omega_{w}\right) \frac{1}{1-\left(2 \omega_{w} V / g \cos \mu_{\circ}\right)}
$$

where $\omega_{w}$ and $\omega_{e}$ are wave and encountering frequencies respectively, $g$ is the acceleration of gravity, $\mu_{\circ}$ is the encountering angle. The spectral density function of the ship response in the irregular sea surface is equal to the product of the spectral density function of waves and the response amplitude operator. Equation (19) is a frequency domain function and can be transformed to a time domain. Wave height and period defining the wave spectrum and a spreading function for shortcrested sea are required.

$$
\begin{gathered}
S_{R}\left(\omega_{e}, \theta\right)=S\left(\omega_{e}\right) \cdot G(\theta) \cdot\left[R A O\left(\omega_{e}, \theta\right)\right]^{2} \\
G(\theta)=\frac{2}{\pi} \cos ^{2} \theta \quad \text { at }|\theta| \leq \frac{\pi}{2}
\end{gathered}
$$



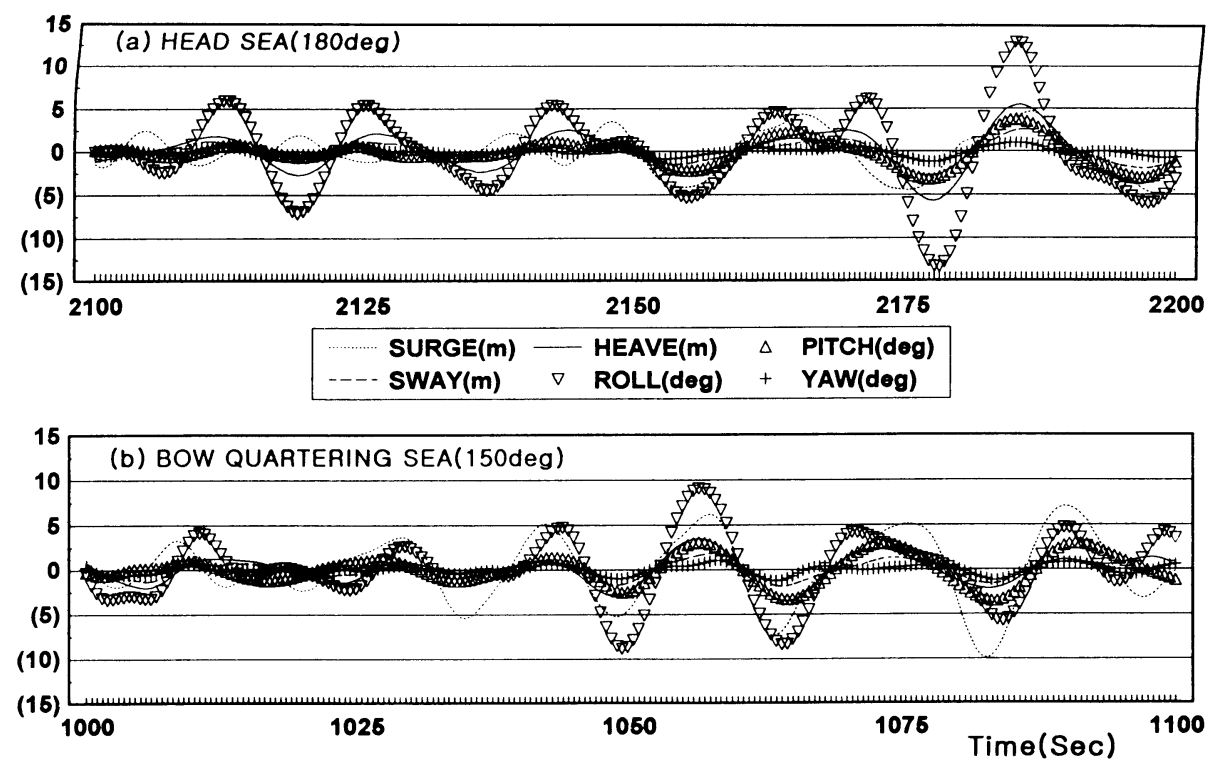

Fig.4 The selected time histories of motions in directional waves for sloshing $\left(V_{\text {ship }}=15.225 \mathrm{Kts}, T_{z}=11.0 \mathrm{sec}\right.$., $\left.H_{1 / 3}=15.75 \mathrm{~m}\right)$

In short-crested waves, the spectral density function $S_{R}\left(\omega_{e}, \theta\right)$ for ship motion can be described as eq. (20) using directional spreading function $G(\theta)$. Equation (20) is the motion spectrum in short-crested waves and can be tranformed to time history data for the ship motion as following,

$$
\eta_{j}(t)=\lim _{n \rightarrow \infty} \sum_{i=1}^{n} \cos \left(\omega_{i} t+\varepsilon\left(\omega_{i}\right)\right) \sqrt{\left.S_{R, j}(\omega, \theta)\right) \delta \omega}
$$

where $j$ means the tank motion of 6 DOF. These time history data of motions are used for the sloshing simulation of LNG carrier in the irregular waves. We considered the time history synthesized by the time histories of ship motions of 6 DOF to produce similar ship motion in irregular waves. The irregular tank motions have been calculated using the wave spectrum with the significant wave height $\left(H_{1 / 3}\right)$ of about 15.75 $\mathrm{m}$ and zero crossing period $\left(T_{z}\right)$ of $11.0,13.0$ and 15.0 seconds. From each hour long tank motion time history, an average record length of about 100 seconds of critical tank motion history was selected for subsequent tank sloshing response calculations. Figure 4 shows the time histories of tank motion in $H_{1 / 3}=15.75 \mathrm{~m}$, $T_{z}=11$ seconds. Severe heave and pitch responses for head sea and bow quartering sea conditions, severe roll and heave responses for beam sea conditions are based

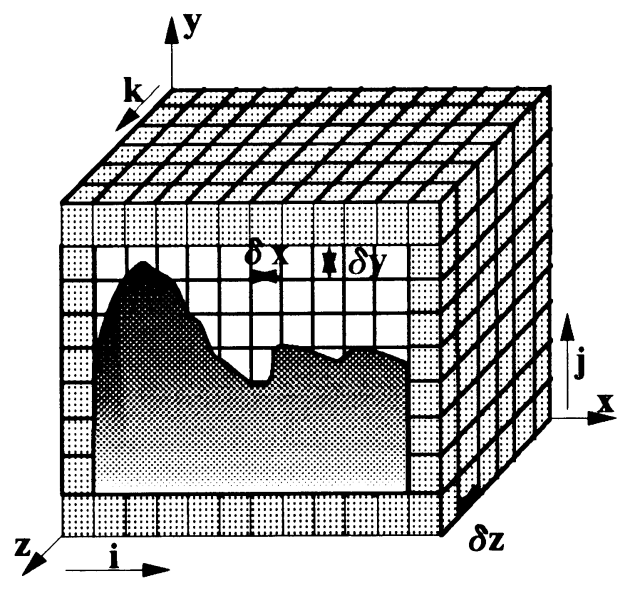

Fig.5 Tank discretization for sloshing calculation

on the selection. Ship motion time histories are used as input data for calculating sloshing simulation in membrane tank of LNG carrier.

\subsection{Results of sloshing calculation}

Sloshing load suimulation method using the 3 dimensional VOF scheme has been developed in this study. For the sloshing simulation, the membrane tanks of 
$138 \mathrm{~K}$ LNG carrier were selected. The ship has four cargo tanks, e.g. No.1, No.2, No.3 and No.4 numbered from bow. Number 2 tank is designated for the estimation of sloshing load due to it's larger dimension among the membrane tanks. The dimension of No.2 tank is shown in Table 1 and No. 2 tank has chamfers in the corner. Filling ratio is selected from the operation load condition of the ship. It is $95 \%$ filling at full load arrival condition. The tank motion for sloshing simulation used the results of ship motion prementioned in section 5.2. The designated sea state is very extreme condition and is taken as the same condition for the structural analysis. The estimated dynamic pressure on the wall of the tank by the sloshing will be used for the direct load analysis of structure. The inside of tank is discretized $29 \times 22 \times 33$ meshes including the boundary wall of membrane tank for the calculation as shown in Fig. 5.

(a)

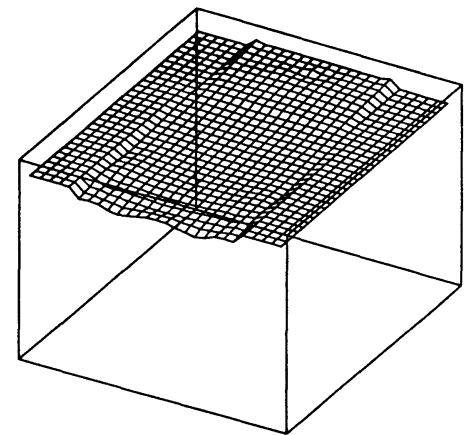

(b)

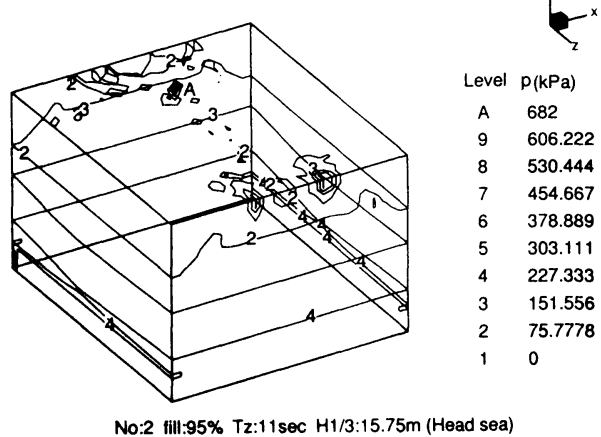

Fig.6 Results of sloshing simulation in directional waves (a) The shape of free surface (b) The pressure distribution(unit ; $\mathrm{kPa}$ )
As mentioned above, the time history data of the estimated ship motion in the directional waves are used for the $3 \mathrm{D}$ sloshing simulation. The calculation is carried out for the cases of head sea and bow quartering sea conditon with the advance ship speed of $15.225 \mathrm{Knots}$. The time history of ship motion in directional waves is estimated during 3000 seconds. Severe motion of 100 seconds, however, is selected from the estimated motion range for sloshing simulation as shown in Fig. 4.

The instantaneous free surface shapes of $95 \%$ filled tank for head and bow quartering sea are shown in Fig.6(a) and Fig.7(a) respectively. The shape of free surface for bow quartering sea looks more realistic than that for head sea because of $3 \mathrm{D}$ effects. The variation of free surface is restricted within the limited wall and

(a)
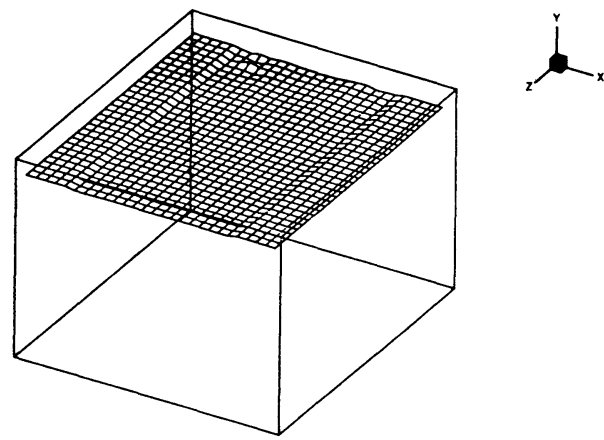

(b)

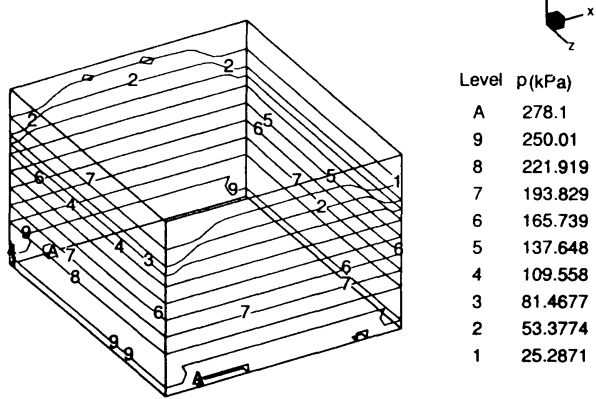

No:2 fill:95\% Tz:11sec H1/3:15.75m (Bow quartering sea)

Fig.7 Results of sloshing simulation in directional waves (a) The shape of free surface (b) The pressure distribution(unit ; $\mathrm{kPa}$ ) 
Table 2: Maximum calculated sloshing pressures $(\mathrm{kPa})$

\begin{tabular}{|c|c|c|c|}
\hline $\mathrm{H}_{1 / 3}=$ & \multicolumn{3}{|c|}{ zero crossing wave periods } \\
\cline { 2 - 4 } $15.75 \mathrm{~m}$ & $11 \mathrm{sec}$. & $13 \mathrm{sec}$. & $15 \mathrm{sec}$. \\
\hline \hline tank & $682(\mathrm{~h})$ & $427(\mathrm{~h})$ & $282(\mathrm{~h})$ \\
\cline { 2 - 4 } capacity & $278.1(\mathrm{q})$ & $278.3(\mathrm{q})$ & $280(\mathrm{q})$ \\
\cline { 2 - 4 } $95 \%$ & $295.3(\mathrm{~b})$ & $295.7(\mathrm{~b})$ & $295.9(\mathrm{~b})$ \\
\hline
\end{tabular}

(h) : head sea

(q) : bow quartering sea

(b) : beam sea

high filling. By these limitation of flow behavior, impact load on the wall and(or) ceiling may not be occurred.

Figure 6(b) and Fig.7(b) show the pressure distribution on the wall and ceiling when the maximum pressure are occurred instantaneously at the selected sea conditions. In the case of head sea, the maximum peak pressure was occurred near the corner at the stern side bulkhead. From the comparison of Fig.6(b) and Fig.7(b), the peak pressure at the bow quartering sea condition is lower than the one at the head sea condition. The maximum peak sloshing pressures calculated are shown in Table 2. In the case of head sea, the impact pressures occurred and peak pressures were inversely proportional to the zero crossing wave periods.

GTT(Gaz Transport and Technigaz) has found that sloshing impact pressures on the flat membrane insulation panel can be reduced by a factor of more than 2.5 when the corrugated membranes are arranged ${ }^{21)}$. The corrugated membrane containment system can provide much greater cushioning effect due to the entraped air than that of the flat membrane. GTT has also indicated that the mean value of the maximum sloshing in MARK III cargo tanks with restricted length and large upper chamfers will not exceed $6.42 \mathrm{kgm} / \mathrm{cm}^{2}(6.3$ bars), with an upper bound of $7.65 \mathrm{kgm} / \mathrm{cm}^{2}$ ( 7.5 bars) and a lower bound of $5.2 \mathrm{kgm} / \mathrm{cm}^{2}(5.1$ bars $)$ from the service experience and the liquid motion test $\left.{ }^{22}\right)$. It is possible that there will be damages on the tank by sloshing as $T_{z}=11$ seconds in head sea, according to the GTT reports. Safe sloshing responses were occurred in beam sea and bow quartering sea and $T_{z}=$ 13,15 seconds. The sloshing peak pressures were more sensitive and larger in head sea than the other seas.

\section{Conclusion}

This study was carried out for developing a numerical code adopting finite difference and SOLA-VOF methods to analyze sloshing flow. The body forces in the governing equations considered the ship motion of 6 DOF and the free surface boundary condition was dealt with numerically as a few steps.

The sloshing analysis in the irregular waves for predicting the largest sloshing responses of $138 \mathrm{~K} \mathrm{LNG} \mathrm{car-}$ rier has been performed for the No.2 tank with fully loaded condition. Random tank motion simulation and tank sloshing peak pressure were discussed. The operating condition that generate the highest sloshing pressure of 6.95 bars is the head sea condition with a wave period of 11 seconds and a filling ratio of $95 \%$. Higher sloshing responses are predicted in tank No.2 of the carrier when it operates severely in head sea than bow quartering and beam sea. Based on a modified pressure peak of 2.78 bars, obtained by reducing the original peak value of 6.95 bars with a factor of 2.5 to account for air cushioning effects, a lower structural responses with an allowable pressure peak values for the case of a corrugated membrane construction of the primary barrier can be expected.

\section{References}

1) Jang. J. H., Hyun. J. K., Ryu. J. H. : Daewoo Shipbuilding Technics, No. 29 (1991), pp.114 121.

2) Soares. C. G. : Loads, 13th ISSC Report Vol.1 (1997), pp. 89 96.

3) Abramson. H. N., Bass. R. L., Faltinsen. O., Olsen. H. A. : Liquid Sloshing in LNG Carriers, 10th Symposium of Naval Hydrodynamics M.I.T. Boston (1974), pp. 371 388.

4) Bass. R. L. : Modeling Criteria for Scaled LNG Sloshing Experiments, Journal of Fluids Engineering, Vol. 107 (1985), pp. 272 280.

5) Cox. P. A., Bowles. E. B., Bass. R. L. : Evaluation of Liquid Dynamic Loads in Slack LNG Cargo Tanks, Southwest Research Institute Report, No. SR-1251 (1979).

6) Tanaka. T. : Estimation of Impact Pressure and Hydrodynamic Force due to Sloshing in LNG carrier, NIPPON KOKAN TECHNICAL REPORT, Overseas, No. 42 (1984), pp. 80.

7) Arai, M., Cheng, L.Y., Inoue, Y. : 3D Numerical Simulation of Impact Load due to Liquid Cargo Sloshing, Journal of Society of Naval Architects of Japan, No. 171 (1992), pp. 177 184.

8) Nagahamai, M., Nagahama, S., Nekado, Y., Yamamori, T., Hori, T. : A 3 Dimensional Analysis of Sloshing by means of Tank Wall fitted Coordinate System, Journal of Society of Naval Architects of Japan, No. 171 (1992), pp. 487 499.

9) Rizzuto. E., Tedeshi. R. : Dynamic Pressures in Sloshing Phenomena ; a Comparison between Full Scale Measurements and Numerical Calculation, Proceedings NAV 94, Session III, Rome. 
10) Hirt. C. W., Nichols. B. D., Romero. N. C. :SOLA Numerical Solution Algorithm for Transient Fluid Flows, Los Almos Scientific Lab. Report LA-5852 (1975).

11) Arai. M. : Experimental and Numerical Studies of Sloshing Pressire in Liquid Cargo Tanks, Journal of The Society of Naval Architects of Japan, No. 155 (1984), pp. 114 121.

12) Mikelis. N. E., Robinson. D. W. : Sloshing in Arbitrary Shaped Tanks, Journal of The Society of Naval Architects of Japan, No. 158 (1985) , pp. 246 255.

13) Shinkai. A., Tamia. S. : Sloshing impact pressure induced on cargo oil tank walls on the middle size double hull tanker, proceedings Trans. Soc. Naval Architects, 90 (1995), pp. 91.

14) Nichols. B. D., Hirt. C. W. : SOLA-VOF; A Solution Algorithm for Transient Fluid Flow with Multiple Free Boundaries, Los Almos Scientific Lab. Report LA-8355 (1980).

15) De Joutte. C., Le Gouez. J. M., Put. O., Rigaud. $S$ : Volume of Fluid(VOF) applied to nonlinear wave problems on body- fitted grids, Proceedings Workshop on Water Waves and Floation Bodies, Hamburg (1996).

16) Cassella. G., Dogliani. M. : Evaluation of Sloshing-induced Fatigue Damage of a FSO tanker, Proceedings 6th IOPE, Los Angeles (1996).

17) Chun. H. H., Kim. M. S., Joo. Y. R. : Seakeeping Analysis of a 30 knots Coastal Passenger SWATH ship, ISOPE, Hawaii (1997), pp. 182 191.

18) Hirt. C. W., Shannon. J. P. : Free Surface Stress Conditions for Incompressible-Flow Calculations, J. Compu. Phys., No. 2 (1968), pp. 403-411.

19) Su. T. C. : A Numerical Analysis of Large Amplitude Liquid Sloshing in Baffled Container, Report No. MA-RD-940-82046, U. S. DOT, Maritime Administration (1982).

20) Raymond. C. Ng. : Advanced Analysis Department Technical Report, AA-97006, ABS AMERICAS (1997).

21) Claude. J., Rico. P. : The cushioning Effect on the Technigaz Membrane, GASTECHM 93.

22) Sloshing Loads in GTT Mark III Membrane Tanks, Gaz Transport and Technigaz External Document No. 1402, Jan. (1997). 